\title{
सितार वाद्य में इटावा घराने की शैलीगत विशेषताएं अरशी
}

शोध छात्रा, संगीत विभाग, पंजाब विश्वविद्यालय, चंडीगढ

\begin{abstract}
Sitar is one of the greatest musical instruments in the India and world. In Indian classical music there are various Gharana (School) of Sitar playing. One of such famous school is Itawa. This school is famous for its unique techniques of Sitar playing. The Alap, compositions (Gatas) of this school are in various talas and varieties of playing techniques are used in the sitar playing.
\end{abstract}

Key words : Sitar, Classical instrumental music, Itawa Gharana.

आधुनिक काल में सितार वाद्य अपनी मधुरता के कारण संगीतज्ञों के अतिरिक्त जन-मानस में भी लोकप्रिय हो गया है। इसकी विकसित वादन शैली तथा वादक तकनीक के कारण इसने भारत में ही नहीं बल्कि पूरे विश्व में अपना स्थान बना लिया है। सितार वाद्य की विभिन्न तकनीकों को इसकी विभिन्न वादन शैलियों द्वारा प्रस्तुत किया जाता है।

19 वीं शताब्दी में सितार वादन के क्षेत्र में भिन्न-भिन्न सितार वादकों की शैलियों की भिन्नता स्पष्ट रूप में दिखाई देने लगी। इन्हीं वादकों व कलाकारों के पुत्रों व शिष्यों द्वारा अपने गुरू की वादन शैली का अनुसरण करने से कालान्तर के कारण सितार वादन के अनेक घराने बन गए। प्रत्येक घराने की वादन शैली की अपनी एक अलग विशेषता थी। सितार वादन के प्रमुख छ: घराने हैं जिनमें से इटावा घराने का सितार वादन की विकसित वादन शैली में विशेष एवं महत्वपूर्ण योगदान है।

इटावा घराने के आदिपुरुष राजपूत गायक 'सरोजन सिंह' को माना गया है जो 18 वीं शताब्दी में इंदौर और रतलाम के मध्य में किसी गाँव में रहते थे। इन्होंने अपनी सांगीतिक शिक्षा उस समय के प्रसिद्ध वीणा वादक 'निर्मल शाह' से प्राप्त की। उनके बेटे 'तुराब खां' "जिनका नाम पहले बद्दू सिंह था" ने धर्म परिवर्तन कर लिया था। ये एक महान संगीतज्ञ थे। तुराब खां के पुत्र साहबदाद खां ही इटावा घराने की अगली पीढ़ी के प्रतिनिधि थे। साहबदाद खां ने ध्रुवपद और ख्याल गायन के साथ सितार और सुरबहार में भी कुशलता प्राप्त की। इनके दोनों पुत्रों में से इमदाद खां ने ही उनकी शैली को आगे बढ़ाया तथा सितार और सुरबहार की एक नयी प्रणाली का प्रतिपादन करने के पश्चात् इटावा घराना एक अलग शेलीगत विशेषताएं समेटे हुए उभर कर सामने आया।

उस्ताद इमदाद खां का जन्म 1848 ई. में इटावा में हुआ। "इमदाद खां इटावा घराने की चौथी पीढ़ी से समबन्धित थे। इमदाद खां को गायन और वादन की शिक्षा अपने पिता से प्राप्त हुई। इमदाद खान ने विकसित दृष्टिकोण तथा कल्पना का संगीत के साथ समन्वय किया। वे सात स्वरों को एक ही परदे पर बड़ी आसानी से और शुद्ध रूप से निकाल लेते थे। उनके साथ ही सितार और सुरबहार वादन की एक नई शैली का प्रतिपादन हुआ जिसे लोग इमदादखानी बाज कहने लगे। 
“इमदाद खान के समय में दाहिने हाथ का काम अधिक होता था अर्थात् मिज़राब के बोल प्रबल थे। इनायत खान के समय में दाएं तथा बांए हाथ का काम एक जैसा था। विलायत खान के वादन में दाहिने हाथ का काम तो वैसा ही रहा लेकिन बाएं हाथ का काम अधिक प्रबल हो गया"1। सितार वादन में गायकी अंग का प्रारम्भ तो शुरू से ही था परन्तु इसके विकास का युग इमदाद खान से प्रारम्भ होकर आज के वादन तक आया है। अगर इमदाद खान का वादन आज सुना जा सके तो स्पष्ट हो जायेगा कि सितार ने अपने गायकी अंग को कितना संवारा है और कितना हृदयग्राही बनाते हुए आज की स्थिति में पहुँचा है। इमदाद खान ने सितार पर तोड़ों का विस्तार किया। तोड़ा, स्वरों और बोलों का जटिल किन्तु व्यवस्थित मिश्रण है। "उस्ताद इमदाद खां ने अपने सितार वादन में विभिन्न बोलों तथा छंदों के मिश्रण से झाले का निर्माण किया। उन्होंने लय की काट-तराश से झाले को महत्व देकर उसे अपने सितार वादन का एक मुख्य अंग बना दिया।"2

"इमदाद खान ने सितार-वादन को समस्त अंगों सहित बजाने की परम्परा डाली जिसमें कलाकार पहले आलाप, जोड़ आलाप एवं जोड़ झाला बजाता था। तत्पश्चात् मसीतखानी और रज़ाखानी बाज की गतें बजाकर झाले के साथ अपने वादन को समाप्त करता था। सितार-वादन के प्रस्तुतिकरण की दृष्टि से सितार-वादन के इतिहास में यह एक महत्वपूर्ण योगदान था।"3

इमदाद खां के पुत्र इनायत खां ने आलाप पर ध्यान दिया जो गत के पूर्व बजाया जाता है। मसीतखानी गत के स्वरूप का मुख्य परिवर्तन उस्ताद इनायत खां के समय से ही आरम्भ हुआ। उन्होंने गतकारी में तिहाई लेने की प्रथा शुरू की। इन्होंने दिर-दिर के बोलों के आधार पर ही तानों का वादन शुरू किया। इसी शताब्दी के सितार में गमक, मींड, कण, मुर्की, कृंतन, ज़मज़मा आदि अलंकरणों का प्रयोग किया गया। इनायत खां साहिब को आधुनिक सितार का जन्मदाता कहा जा सकता है। इन्होंने परम्परागत सितार-वादन में अनेक सुधार करके एक अभिनव वादन शेली को प्रचारित किया। इनके पश्चात् उस्ताद विलायत खां साहिब ने इस घराने की वादन शैली को अपने ढंग से सजाया एवं संवारा है। "विलायत खां साहिब ने सितार वादन को एक नई दिशा दी। उनकी सितार में गायकी अंग के अनुसार आलाप, जोड़, गत यानी सब चीजें गायन के रूप में, न कि वादन के रूप में बजती हैं। यह अंग सितारियों को इतना भाया , कुछ को छोड़कर अधिकतर सब ने अपना लिया।"4

उस्ताद विलायत खां आलाप को स्थायी, अंतरा, संचारी और आभोग में विभक्त करके बजाते थे। विलायत खां के आलाप की विशेषता विलोम मींड तथा एक ही आघात में कई स्वर उत्पन्न करने की थी। वैसे अनुलोम मींड को भी बजाते थे लेकिन विलोम मींड बजाना अधिक कठिन है जिसको

1 डॉ. कविता चक्रवर्ती, उस्ताद विलायत खान का घराना एवं सितार वादन शैली, लेख, संगीत, दिसम्बर 2001, पृ. 46.

2 डॉ. वी. एस. सुदीप राय, जहान -ए-सितार, पृ. 69

3 रजनी भटनागर, सितार वादन की शैलियाँ, पृ.182

4 शान्तनु शर्मा, प्रतिष्ठित सितार और सरोद वादकों की साधना और संघर्ष, पृ. 83. 
उन्होंने बड़ी मेहनत से हासिल किया था। "विलायत खां साहिब का कहना है कि बीन में जोड़ अंग काफी बजता था। अतः सितार में भी बीन अंग के प्रभाव से ही जोड़ बजने लगा।"1

विलायत खां जब जोड़ प्रारम्भ करते थे तो प्रारम्भ में लय उभरकर आती थी, मध्य लय में खां साहिब लय थोड़ी बढ़ाकर तोड़े बजाते थे और फिर झाला बजाते थे। झाले में खां साहिब चिकारी की तार पर 'रा' और बाज की तार पर 'दा दा दा' बोल बजाते थे।

$$
\begin{aligned}
& \text { जैसे: - } \\
& \text { रा दा दा दा }
\end{aligned}
$$

"विलायत खां साहिब का कहना है "मैंने सितार पर ख्याल गायकी का आरम्भ किया है जिसका आज के सितार वादकों द्वारा अनुसरण किया जा रहा है। मैंने पंजाबी अंग में ठुमरी शैली के संगीत को समृद्ध करने का प्रयत्न किया है। लोकधुनें भी अपनाई हैं। पंजाबी अंग की ठुमरी शेली के बारे में उन्होंने बताया कि पहले इसका भिन्न रूप और ताल थी और अब सितार पर ठुमरी-वादन पूर्णतः इसकी गायन शैली पर आधारित है जिसमें दीपचन्दी तथा चंचल तालों का प्रयोग होता है।"2 इन्होने ही बाएं हाथ की तकनीक का आरम्भ किया जिनमें एक स्वर के लम्बे अन्तराल की निरन्तरता प्राप्त हुई। उन्होने इस तकनीक का प्रयोग गायन शेली का निकटता से अनुसरण करते हुए भावकाव्यात्मक के प्रकल्प के लिए किया। इसके साथ ही उन्होंने उच्च, जटिल व कठिन ख्याल अंग की मुर्कियों का आरम्भ किया। इनके सितार वादन में शुद्धता उनकी कला की विशेषता है।

उस्ताद विलायत खां ने अपने घराने की मर्यादा का पालन करने में अपने कर्तव्य-परायणता का पूरा परिचय दिया है। वर्तमान समय में प्रबल इच्छा शक्ति के बल पर उच्चतम स्तर पर पहुँचने वाले संगीतज्ञ एवं प्रसिद्ध सितार वादक उस्ताद विलायत खां साहिब ने सितार वाद्य की जिस वादन शैली का विकास एवं वादन में माधुर्य तथा नवीनता पैदा की है, वह वास्तव में एक नई क्रान्ति के रूप में जानी जाती है। "इस घराने के कलाकारों में ठुमरी बजाने की परम्परा भी रही है। इमदाद खां स्वयं विभिन्न तालों में ठठमरियाँ रचा करते थे।"3

इनायत खां साहिब के बारे में कहा जाता है कि "ठुमरी अंग की चीज़ सितार पर बजाने में वे सिद्धदस्त थे। उनका वादन सुनकर श्रोताओं के अन्दर से 'वाह-वाह' स्वतः ही उच्छ्वासित हो जाती।"4 विलायत खां भी अपने सितार वादन के अंतर्गत् ठुमरी बजाते थे। सितार पर ठुमरी बजाने के साथ-साथ तराना बजाने की परम्परा भी इस घराने के कलाकारों में रही है। खां साहिब अपनी वादन विधि में तराना बंदिश को भी बजाते थे।

1 डॉ. कविता चक्रवर्ती, उस्ताद विलायत खान का घराना एवं सितार वादन शैली, लेख, संगीत, दिसम्बर 2001, पृ. 47.

2 सत्येन्द्र शर्मा, उस्ताद विलायत खान, लेख, संगीत, मार्च 1961, पृ. 44

3 रजनी भटनागर, सितार वादन की शैलियाँ, पृ. 182

4 जितेन्द्र मोहन सेन, उस्ताद इनायत खान साहिब के साथ 10 वर्ष, लेख, संगीत, अगस्त 1994, पृ.17 
वर्तमान समय में इसी घराने की परम्परा के वाहक, सितार वादक उस्ताद शाहिद परवेज़ खां श्रेष्ट सितार वादकों में गिने जाते हैं। इन्होंने अपनी घरानेदार तालीम अपने दादा उस्ताद वहीद खां ताया उस्ताद हफीज़ खां तथा पिता उस्ताद अज़ीज खां से प्राप्त की। ये इटावा घराने के सातवीं पीढ़ी के प्रतिनिधि कलाकार हैं, जिन्होने अपने पिता की कथनी को करनी कर दिखाया। इनका सितार वादन गायकी अंग और तंत्रकारी अंग के मिश्रण पर आधारित है।

साहबदाद खां से लेकर वर्तमान समय तक इस घराने ने सबसे अधिक सुप्रसिद्ध एवं उत्कृष्ट कलाकार दिए हैं, जिनमें इस घराने के वंशज और शिष्य भी हैं। इन्होंनें इस घराने को अग्रसर किया तथा अपने दृढ़ संकल्प और अपनी सांगीतिक साधना से इस घराने को शिखर तक पहुँचाया।

संदर्भ ग्रन्थ सूची

गर्ग, लक्ष्मी नारायण (1977) संगीत निबंधावली, संगीत कार्यालय, हाथरस, उत्तर प्रदेश, तृतीय आवृति। भटनागर, रजनी (2006) सितार वादन की शैलियाँ, कनिष्क पब्लिशर्स डिस्ट्रीब्यूटर्स, नई दिल्ली। राय, वी.एस. सुदीप (2004) जहान-ए-सितार, कनिष्क पब्लिशर्स डिस्ट्रीब्यूटर्स।

शर्मा, शान्तनु (2005) प्रतिष्ठित सितार एवं सरोद वादकों की साधना और संघर्ष, कनिष्क पब्लिशर्स डिस्ट्रीब्यूटर्स, नई दिल्ली।

हिन्दी पत्र-पत्रिकाएँ

गर्ग, लक्ष्मी नारायण, संगीत, संगीत कार्यालय हाथरस, उत्तर प्रदेश, संस्करण-मार्च 1961।

गर्ग, लक्ष्मी नारायण, संगीत, संगीत कार्यालय हाथरस, उत्तर प्रदेश, संस्करण-अगस्त, 1994।

गर्ग, लक्ष्मी नारायण, संगीत, संगीत कार्यालय हाथरस, उत्तर प्रदेश, संस्करण-दिसम्बर, 2001 । 